\title{
ANTITUMOR ACTIVITY OF BIOMOLECULES: A BRIEF REVIEW
}

\author{
SOUZA P.O. ${ }^{1}$, RITTER M. ${ }^{1}$, BRAGANHOL E. ${ }^{2 *}$ AND PEREIRA C.M.P. ${ }^{1}$ \\ 1Laboratory of Lipidomics and Biorganic - LLipidomicaBio, Federal University of Pelotas, Pelotas, RS, Brazil. \\ 2Laboratory of Neurochemistry, Inflammation and Cancer - NeuroCan, Federal University of Pelotas, Pelotas, RS, Brazil. \\ ${ }^{*}$ Corresponding Author: Email- elizbraganhol@yahoo.com.br
}

Received: March 28, 2014; Accepted: May 15, 2014

\begin{abstract}
Cancer is one of the major public health problems at the world. The present review explores the cancer hallmarks and the basis of tumor progression, showing some biological characteristics, and how the immune system reacts to it. Half of drugs that are in clinical use today are of natural origin, indicating that natural products have a significant role in the process of discovering and developing drugs as prototypes. From this understanding, the present review will show some biological molecules, including heterocyclic compounds that have potential activity against cancer.
\end{abstract}

Keywords- Active biologically molecules, Cancer, Drugs, Heterocyclic compounds, Immunological activity, Natural products.

Citation: Souza P.O., et al (2014) Antitumor Activity of Biomolecules: A Brief Review. International Journal of Drug Discovery, ISSN: 0975-4423 \& E-ISSN: 0975-914X, Volume 6, Issue 1, pp.-213-223.

Copyright: Copyright@2014 First Author, et al. This is an open-access article distributed under the terms of the Creative Commons Attribution License, which permits unrestricted use, distribution and reproduction in any medium, provided the original author and source are credited.

\section{Developments in Cancer Treatment}

Although the idea that cancer is a modern disease caused by current life style, there are references of this disease in ancient Greece and Egypt. Histological studies of mummies found in Chile and Egypt showed the presence of benign neoplasms. This theory is based in the concept that the genetic mutations accumulated throughout evolution may result in cell abnormalities and lead to tumor development $[1,2]$.

Hippocrates, considered medicine's father (460-370 B.C.) introduced the terms carcinos and carcinoma to describe certain types of tumors, which in Greek mean crab. This name was given based on appearance of the tumor, since the projections and blood vessels around her remind the legs of the crustacean. Some centuries later, Galleno continued the Hippocrates's legacy and postulated cancer as an incurable and untreatable disease [3].

For a long time the cancer treatment was restricted to the use of plants and therapeutic surgery [4]. In the early twentieth century, radiation therapy, which was based on the principle that the radiation was selective to tumor cells, while the surrounding normal cells were unaffected, was introduced to medical practice. However, the clinical data indicate that the radiation therapy is deleterious to normal tissues and high precision equipment to destroy the tumor without damaging the normal cells was developed to increase the treatment efficacy and reduce the side effects of radiation [5].

Following World War II emerged chemotherapy drugs for cancer treatment, which has the function to impair cell mitosis, affecting the fast-growing cells. Thus, in addition to target cancer cells, this treatment also affects the cells responsible for hair growth and replacement of the epithelium gut wall [6].
Currently, depending on the tumor type and the severity of the situation, these treatments are combined to have greater effectiveness against cancers presented by the patient thereby allowing a greater survival and life quality after the development of the disease.

\section{Characterizing Tumor}

Tumor cells or neoplastic cells, among other characteristics, accumulate mutations in several genes related to cell proliferation, death, migration which result in sustaining proliferative and angiogenesis signaling, resistance to cell death and to chemotherapy, activation of invasion and metastasis [7]. Histopathological characteristics reveal that tumor mass architecture is less organized and structured when compared to normal tissues. This observation contributes to conception that tumors are composed by cells that have lost their ability to create tissues with normal form and function, arising from a complex cascade of phenotypic changes followed by uncontrolled growth [8].

The tumors were divided into two general categories according to its degree of aggressiveness: those located growth without invasion of adjacent tissues have been classified as benign while the tumors that invade nearby tissues and spread of metastasis were called malignant [7]. Note that some benign tumors can cause clinical problems by releasing high levels of hormones that cause physiological imbalances in the body, including spinal and paraspinal tumors that releases catecholamine among others chemicals resulting in diarrhea and hypertension [9].

Hanahan \& Weinberg [10] outlined six essential characteristics acquired by mutation, which define a cell as neoplastic: selfsufficiency in growth signals, lack of response to external signaling 
antiproliferative, unlimited proliferative capacity, ability to promote angiogenesis, tissue invasiveness and metastasis and escape or resistance to apoptosis. New enabling added two potential hallmarks to this list, those are reprogramming of energy metabolism and evading immune destruction. Another relevant discovery is that tumors exhibit other dimension of complexity: they contain a set ostensibly normal cells that contribute to the acquisition of hallmark traits by creating the "tumor microenvironment" [11].

Oncogenes and anti-oncogenes are genes that participate in the formation of tumors. The anti-oncogenes or tumor suppressor genes are recessive, that is, the carcinogenic effect appears only when they are missing or defective in both chromosomes of the genome. In contrast, oncogenes are dominant and encode proteins related to disordered cell multiplication, becoming malignant. Just a copy of the oncogene in the genome promotes the transformation of normal cells into cancer cell. Proto-oncogenes are growth factorrelated genes, which regulate normal cell differentiation and proliferation. They encode growth factors, membrane receptors and DNA binding proteins [12].

\section{Signaling Pathways and Growth Factors}

There is considerable interest in understanding how activated signaling pathways enhance tumor cell survival because this may lead to the introduction of more effective treatments to target the chemoresistant cell subpopulations [13]. As signals are propagated along the cancer pathways by a relay of protein kinases, developing specific protein kinase inhibitors to target particular pathways may constitute novel therapeutic strategies for cancer intervention [14].

In general terms, the core of signaling pathways included those in which a single, frequently altered gene predominate, such as KRAS signaling and regulation of G1/S cell cycle transition; pathways in which a few altered genes predominated, such as TGF- $\beta$ signaling; and pathways in which many different genes were altered, such as adjusting of invasion process, homophilic cell adhesion, and integrin and small guanine triphosphatase (GTPase) - dependent signaling. In addition, JNK (c-Jun N-terminal kinase) signaling, Wnt/Notch signaling, Hedgehog signaling, apoptosis and DNA damage control constitute pathways altered in cancer cells [15].

A genetic analysis of pancreatic cancer showed 12 cell signaling pathways that are genetically altered in $67-100 \%$ of these tumors [15]. Mutations in the genome of cancer cells affect signaling pathways, which develop a crucial role in cell growth, proliferation, metastasis, angiogenesis, survival and apoptosis. Activation of these signaling pathways leads to up-regulation of a group of transcriptional factors that induce epithelial-to-mesenchymal transition in cells [16]. Some signaling pathways are essential for embryonic development that has critical roles in the variation in tumor progression and response to therapy in a variety of human cancers, such as Hedgehog and Wnt pathways. The activation of several pathways and their interactions also raise difficulties in overcoming chemoresistance [14].

PI3K (phosphatidylinositol 3-kinase)/Akt signaling pathway is considered as the most important pathway involved in modulation of tumor survival and metastasis, that is activated by various growth factors and also by non-canonical pathways, including activation by various (proto-)oncogenes such as Ras, Her2/neu, cKIT [17]. A variety of reports have demonstrated in various cell types that the constitutive activation of Akt (protein kinase B) signaling is sufficient to block cell death induced by a variety of apoptotic stimuli and that the transduction of dominant-negative Akt inhibits growth factorinduced cell survival [18].

The PI3K/Akt signaling pathway may be associated with the motility and migration ability of metastatic tumor cells. Increasing PI3K signaling in cancer is triggered to some mechanisms, among them tyrosine kinase receptors activation and somatic mutations or amplification of genes encoding key components of the signaling cascade [17].

The embryonic signaling pathways, Hedgehog, Notch and Wnt, are activated in the majority of cancers. These pathways are critical for the cellular development processes and regulation of normal stem cells [19].

Hedgehog $(\mathrm{Hh})$ signaling modulates tissue polarity, morphogenesis, proliferation and differentiation and is responsible for stem cell maintenance [16]. This pathway is activated in nearly every type of cancer, as glioblastoma, basal cell carcinoma, pancreatic cancer and breast cancer, and is important in maintaining the cancer stem cell in different hematological and solid malignancies, contributing to cancer proliferation, progression and invasiveness [20]. The $\mathrm{Hh}$ pathway is deregulated differently depending on the tumor type, with abnormal activation occurring either in a ligand-independent or a ligand-dependent manner [19].

Notch signaling is important for cell-cell communication, cell fate specification, cell proliferation, and apoptosis both in embryonic development and in adult tissues. Notch deregulation has been implicated in oncogenesis [21]. Notch signaling is clearly contextdependent and its activation can result either in pro- or antioncogenic effects. The pro-tumorigenic outcome is based on antiapoptotic, cell growth and angiogenesis induction, and EMT facilitating effects [19].

Wnt signaling plays a key role in several biological aspects, such as cell proliferation, tissue regeneration, embryonic development, and another systemic effects recently discovered, such as bone biology, auto-immune diseases, neurological diseases, neoplastic disorders, angiogenesis and vascular disorders [22- 25]. The Wnt pathway has been well studied in a number of cancers that exhibit mutations on $\beta$-catenin $[26,27]$. According to Rosenbluh and coworkers emerging evidence indicates that oncogenic $\beta$-catenin regulates several biological processes essential for cancer initiation and progression [26].

The Fas/FasL signaling plays a significant role in tumorigenesis. Fas is a membrane receptor belonging to the TNF receptor (TNFR) superfamily, which ligand is FasL. Since Fas/FasL play an important role in extrinsic pathway of apoptosis, alterations in this protein contribute to apoptosis resistance and consequent tumor progression [28].

TGF- $\beta$ signaling also plays a significant role in EMT process, embryogenesis, and cancer pathogenesis. TGF- $\beta$ can alter tight junction formation in mammary epithelium and controls a number of embryonic signaling pathways, including Wnt, Notch and Hh pathways. This signaling can inhibit or suppress transcription of Ecadherin, occludin and claudinin in order to initiate cancer growth and metastasis [16].

Over two decades ago, VEGF (vascular endothelial growth factor), which was initially discovered as vascular permeability factor, was identified as the main regulator of tumor angiogenesis. The role VEGF receptor 1 (VEGFR1) in tumor angiogenesis is complex and still not completely understood. VEGFR1 is involved in the regula- 
tion of vascular development during embryogenesis and the recruitment of endothelial cell progenitors. Thus, the inhibition of VEGF results in the successful suppression of tumor growth [29].

Epidermal growth factor receptors (EGFR) are a large family of receptor tyrosine kinases (RTK) expressed in several types of cancer, including breast, lung, esophageal, head and neck [30]. EGFR and its family members are the major contributors of a complex signaling cascade, among the main features stand out adhesion, differentiation, growth modulate, migration and survival of cancer cells [31]. EGFR signaling activation can stimulate the synthesis and secretion of a number of angiogenic-regulating factors, such as VEGF, Interleukin-8 (IL-8) and basic fibroblast growth factor (bFGF). It is well known that phosphorylation of EGFR is essential for EGFR signaling, which results in phosphorylation at tyrosine residues on the intracellular domain of the receptor, thereby triggers the corresponding signaling pathways [32].

The insulin-like growth factor (IGF) system controls cell growth, differentiation, and development. IGF1 receptor (IGF1R) is a tyrosine kinase receptor with $60 \%$ homology to the insulin receptor (IR), which signaling is deregulated in many cancers [33]. IGF1R exerts anti-apoptotic effects and plays a role in cancer cell proliferation and motility, supporting the mitogenic and metastatic role of such molecule [34].

The MAPK pathway includes some key signaling components and phosphorylation events that play important role in tumorigenesis. When activated, these kinases transmit extracellular signals that regulate cell growth, differentiation, proliferation, migration and apoptosis functions. Alteration of RAS-RAF-MEK-ERK-MAPK (RAS -MAPK) pathway was observed in human cancer as a result of abnormal activation of receptor tyrosine kinases or gain-of-function mutations mainly in the RAS or RAF genes [35].

\section{Programmed Cell Death}

In a multicellular organism, cell exposure to a set of environmental factors may activate specific intracellular programs that could lead to the cell morphological changes and ultimately to cell death [36]. These cell-killing intracellular events constitute the programmed cell death phenomenon, which includes apoptosis, autophagy, necroptosis (programmed necrosis) and pyroptosis [37].

Programmed cell death may balance cell death with survival of normal cells; when the equilibrium becomes disturbed, programmed cell death plays key roles in ultimate decisions of cancer cell destination [11]. It is well known that cancer cells have numerous strategies to overcome programmed cell death [38].

Apoptosis is characterized by retraction of pseudopodes, the rounding up of cells and the detachment from the basal membrane or cell culture substrate; a consistent decrease in cell volume (pyknosis); chromatin condensation and nuclear fragmentation (karyorrhexis); blebbing of the plasma membrane; shedding of vacuoles containing parts of the cytoplasm and apparently intact organelles (the socalled apoptotic bodies); and in vivo uptake of apoptotic corpses by neighboring cells or professional phagocytes [39]. The major mechanism that actually causes cell to die is associated with the organized degradation of cell organelles by activated members of caspase family of cysteine proteases [11].

Apoptotic signals exhibit two main pathways connected to each other. The activation of cell-surface receptors induces the extrinsic pathway and the intrinsic pathway starts with stress signals [11].
The mitochondrial pathway is an intrinsic pathway to induce apoptosis in which the cell becomes initiated by either extracellular stimuli or intracellular signals, outer mitochondrial membranes become permeable to internal cytochrome $\mathrm{c}$, which is then released into the cytosol [38]. Cytochrome c recruits Apaf-1 and pro-caspase-9 to compose the apoptosome, which downstream triggers a caspase 9/3 signaling cascade, leading to induction of apoptosis [40].

Transcriptionally independent activity of p53 can potentiate the apoptotic response in a direct interaction with members of the BCL2 family of proteins with structurally conserved domains, which have an important role in positively and negatively regulating mitochondrial apoptotic pathway, allowing p53 to function as a so-called $\mathrm{BCL}$-homology domain-3 (BH3)-only protein. Two of these $\mathrm{BH}-$ domain proteins, BAK and BAX, promotes apoptosis regulating mitochondrial membrane potential [41].

According Vousden and Lane [41], one of the key contributions of p53 to apoptosis is the induction of the expression of genes that encode apoptotic proteins, which can use intrinsic and extrinsic pathways. Many of these genes of p53 were reported, including those that encode the BH3-domain proteins NOXA and PUMA.

Autophagy mediates the turnover of long-lived proteins, the elimination of damaged organelles and misfolded proteins, and the recycling of cell building blocks following nutrient deprivation. Autophagy has a crucial pro-survival role in cellular homeostasis and during stress, but under certain circumstances may commit suicide by undergoing cell death [40]. Stress is a common property of tumors; those have hypoxic regions and probably also growth factors and nutrients due to abnormal or insufficient vasculature [42]. So, autophagy can have either pro-survival or pro-death roles [36].

At extreme conditions as when a cell is starving, it is well known that proteins related to autophagy are initiated [43]. Among these proteins, microtubule associated process 1 light chain 3 (LC3), mammalian autophagy protein, is a key regulator involved in forming autophagosomes. Autophagosome marker (LC3) exists in three isoforms (LC3A, LC3B and LC3C) [44]. During autophagy LC3 is cleaved to its cytosolic form LC3-I. Lipidation leads to the LC3-II form, which becomes associated with the autophagosomes. Conversion of LC3-I to LC3-II is therefore a marker for the autophagosome formation, which fuses with the lysosome membrane, then their contents degraded by the lysosomal enzymes [43].

Studies have reported high levels of expression of LC3 in some kinds of cancers as pancreatic, esophageal, colorectal and gastric, suggesting that autophagy is closely associated with tumor [43,4546]. However, increased LC3 expression can't accurately reflect increased autophagic activity; it can also indicate a decrease in autophagic function as a result of a block in fusion after increases in the numbers of autophagosomes [47].

In addition, according Cui and colleagues cellular stress can induces autophagy, consequently, promotes the growth and survival of cancer cells, so facilitates tumorigenesis and results in resistance to therapy [48]. Inhibition of autophagy has been shown to sensitize tumor cells to the cytotoxic effects of chemotherapy and ionizing irradiation to enhance cancer treatments [49].

In contrast to apoptosis, in which a dying cell contracts into an almost-invisible corpse that is soon consumed by nearby cells; necrotic cells become turgid and explode, after that their contents are released into the local tissue microenvironment. Thus, necrotic cell death, in contrast to apoptosis and autophagy process, releases pro 
-inflammatory signals into the surrounding tissue microenvironment [38].

Programmed necrosis or necroptosis is an alternative form of programmed cell death that depends on the serine/threonine kinase activity of RIP1 [50]. The permeabilization of the plasma membrane can be the cause of later cell death due to ruptures caused by cytoplasmic swelling and consequent release of cytoplasmic contents. This feature is share with pyroptosis, another kind of cell death [51]. In most cases, necroptosis is initiated by stimulation of the extrinsic apoptotic pathway when caspases are absent or inhibited, in other words, when apoptosisis blocked, necroptosis becomes the predominant form of cell death. Increased ROS levels are a hallmark of necroptosis and may be one of the main causes of necroptotic cell death [50].

Altogether, self-destruction can occur also from inside cellular environment [36]. Accordingly, genetic damage leads to p53 activation, that induces cell cycle arrest and DNA repair, or programmed cell death, an extreme and radical means preventing the emergence of genetic heterogeneity, and the progression towards cancer [11]. Similarly, changes in endoplasmic reticulum integrity, induced by abnormal protein folding, or alterations in mitochondrial activity, such as respiratory chain dysfunction can result in signaling leading to programmed cell death [50]. Thus, cell suicide is responsible for the maintenance of the genetic identity and the integrity of the body, once that induces fast elimination of altered cells.

\section{Immune Defense}

One of the most important response mechanisms to DNA damage involves the p53 tumor suppressor, the so-called "guardian of the genome" [41]. This tumor suppressor is one of the most important and extensively studied, it influences a lot of cellular processes. Indeed, genomic and mutational analyses documenting inactivation of p53 in more than $50 \%$ of human cancers motivated drug development efforts to (re-) activate p53 in established tumors [52].

It is well known that the immune system is able to mount responses against tumors and that this effect can be enhanced using a number of strategies [53]. The interplay between tumor cells and immune system is complex. The concept of cancer immune surveillance has been proposed more than one century ago, but has only recently been validated. According to this concept, immune cells repress tumor growth [54].

Immune surveillance is the first step of the interactions between immune system and tumor cells, corresponding to the tumor elimination phase. If there are only few cancer cells, the immune system can eradicate the tumor at this early stage. If immunosurveillance fails to eradicate the tumor, a second step called the equilibrium phase can be observed. At this stage, tumors are not clinically detectable and the immune system only constrains tumor cell growth. Tumor cells that develop mechanisms to evade the immune system allow cancer to become immune resistant in a process known as cancer immune editing. Then, the tumor cell mass increases and becomes clinically detectable [54].

Many elements are involved in the immune surveillance process. Tumor antigens can be presented by Major Histocompatibility Complex (MHC) molecules to the antigen-presenting cells (APCs). The anti-tumor immune response involves both innate effector cells such as Natural Killers cells (NK), Dendritic Cells (DCs), macrophages, granulocytes and proteins of complement, and adaptive immune responses mediated by $B$ and $T$ lymphocytes [55].
Immune cells can be activated by multiple mechanisms. Tumor antigens can be presented by MHC molecules, activating a tumorspecific response. NK cells can be activated by the decreased expression of MHC class I molecules on cancer cells. In addition, immune cells can be activated by the overexpression of activating ligands on cancer cells [56]. Then, mucins, glycoproteins in mucus are frequently overexpressed in diverse cancer cells and are involved in chronic inflammation, oncogenesis, survival, tumor growth and invasion pathways. It has been shown that MUC1 mucin could inhibit tumor cell lysis by NK cells. This approach reveals mucins as a potential therapeutic target in oncology [57].

Tumor antigens can be presented to lymphocytes by APCs, mainly DCs, macrophages and CD4+ lymphocytes. T-cell activation requires the recognition by the T-cell receptor of the tumor antigens presented by MHC products. This activation also requires additional signals provided by the APC, e.g. generated through the interactions between $B 7$ and $C D 40$ ligand expressed on the $T$ cell, with CD28 and CD40, respectively, expressed on the APC. CD28 stimulation induces the expression of CTLA-4 on the T-cell membrane. CTLA-4 will interact with B7 with a greater avidity than CD28, and will inhibit the activated T cell [56].

Tumor-infiltrating lymphocytes (TILs) are located both within the tumor and in the peritumoral stroma. Main subsets of TILs are NK lymphocytes, $\mathrm{CD} 8^{+}$cytotoxic lymphocytes, $\mathrm{CD} 4^{+}$helper lymphocytes and $\mathrm{CD} 45 \mathrm{RO}^{+}$memory $\mathrm{T}$ cells. They can play a role in inhibition and control of the immune response (CD25+ and FOXP3+regulatory $T$ cells) [56].

It has been demonstrated that many malignant tumors have high infiltration of macrophages. Macrophages at the tumor periphery can foster local invasion by supplying matrix-degrading enzymes such as metalloproteinases and cysteine cathepsin proteases [58]; in a model system, cancer cells produce IL-4 that activate macrophage invasion [59].

These macrophages, also known as tumor-associated macrophages (TAMs), have been implicated in stromal activation, invasion, and metastasis. TAMs have been shown to regulate angiogenesis and tumor growth by producing potent cytokines and growth factors [59]. One of hallmarks of cancer allows cancer cells to evade immunological destruction, especially by macrophages, B and T lymphocytes, and natural killer cells [11].

Necrotic cells release regulatory factors, such as IL-1a, which can directly stimulate neighboring viable cells to proliferate, with the potential, once again, to facilitate neoplastic progression [60].

According cancer immunology, simplifying tumor-host immunological interactions, as highly immunogenic cancer cells may well evade immune destruction by disabling some components of the immune system that have been send to eliminate them [11]

\section{Biomolecules with Antitumor Activity}

Natural products, especially those of terrestrial plants and microorganisms, have been exploited as a traditional source of molecules for pharmaceuticals; beyond its pharmacologically active compounds are important for further investigations [61].

The first plant compound against cancer was discovered in the bark, and at low levels in the needles, of the relatively rare Pacific Yew, Taxus brevifolia. In the 1970s, taxol, recently named paclitaxel, was discovered [Fig-1]. Taxol become one of the most effective drugs against breast and ovarian cancer and has been approved worldwide for the clinical treatment of cancer patients [4]. This find- 
ing represented significant advances in cancer therapy, paclitaxel has a well-known mechanism that blocks cell mitosis and kills tumor cells [62]. In the 1990s, Robert A. Holton published the first total synthesis of paclitaxel [63].

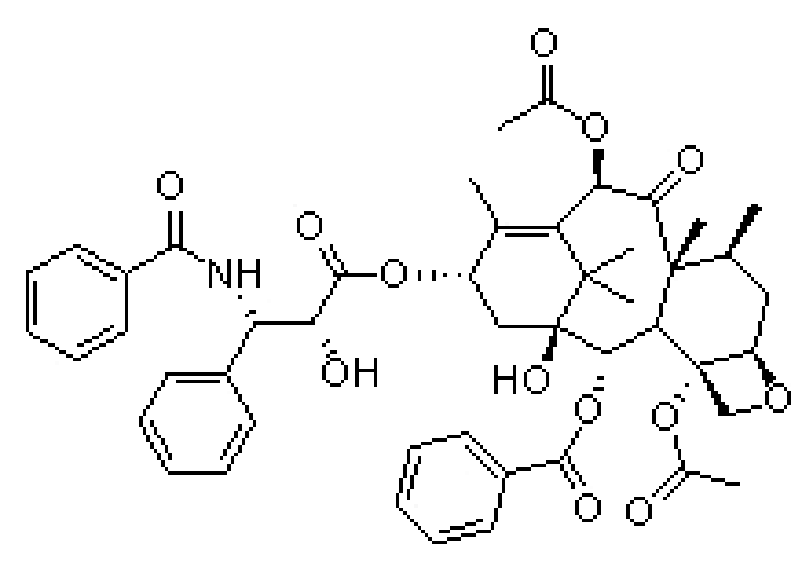

Fig. 1- Structure of taxol.

Biosynthesis of carbohydrates is a basic process of life and quantitatively the most important. They are constituent parts of complex lipids (glycolipids) and proteins (glycoproteins) as well as nucleotide building blocks, consequently by nucleic acids and the chemical energy storage system of ADP/ATP [64]. Due to the inherent properties of this class of molecules, carbohydrates have been used to prepare bioactive materials [65], and drugs to specific targets [66].

The complexion of carbohydrates to metals was assessed by Sathisha and coworkers [64] who associated N-glycosyl with chloride Co (II), Ni (II), Cu (II) and Zn (II) [Figs-2,3,4] noting that the compounds significantly reversed $(p<0.05)$ in tumor-induced changes in the model of Ehrlich ascites carcinoma. All compounds showed significant cytotoxicity in cell viability test, $25 \%$ increase in the lifetime of the animals. By convention, a test compound showing $25 \%$ increase of the lifetime is considered possible with anticancer activity [65].

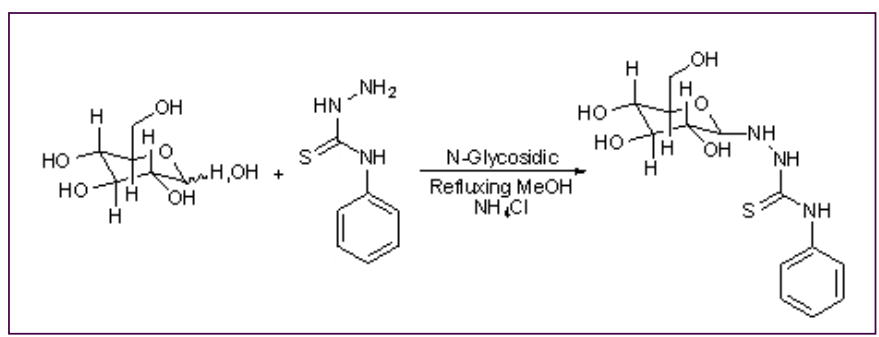

Fig. 2- Synthesis of (D-glucopyranose)-phenylthiosemicarbazide $\mathrm{LH}$.

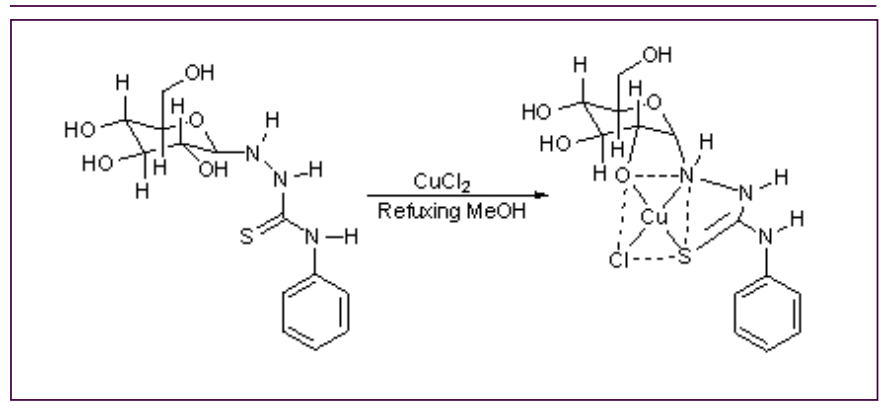

Fig. 3- Synthesis of [CulCl].

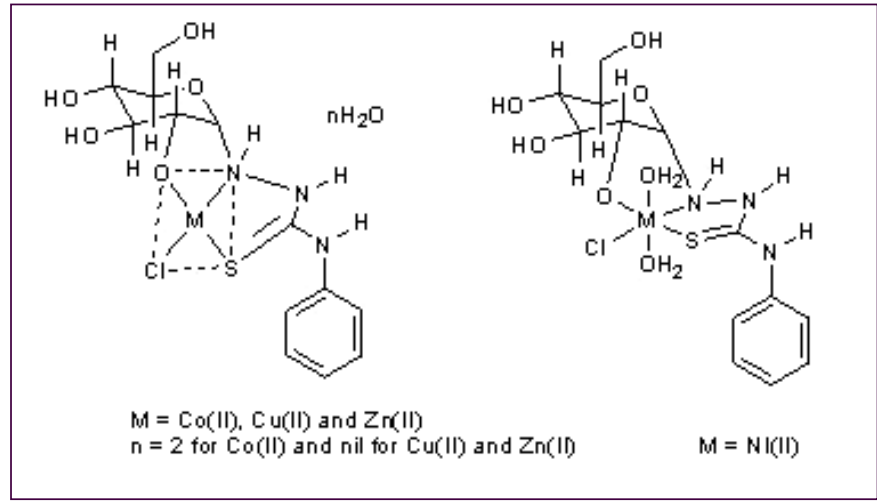

Fig. 4- Synthesis of Cobalt(II), Nickel(II), Copper(II) and Zinc(II) complexes.

Polysaccharides are widely present in many plants and have numerous biological activities, being increasingly used in medicines [68]. The biological activities of polysaccharides depend on the sugar moiety, glycosidic linkages in the main chain, the type and degree of polymerization of the branches, flexibility and configuration of the chains, which can be modified as a polymer by chemistry derivatization [69].

Modification in the structure of the polysaccharide is an effective way to increase the biological activities of the polysaccharide. Sulfated polysaccharides, including both naturally extracted from plants as derivatives synthesized are types of biomolecules with sulphated groups in their hydroxyl groups and have different or stronger biological activities, among them antitumoral [70].

Thus, Wei and coworkers [68] extracted a polysaccharide from Radix hedysari (RHP), which is the dried root of Hedysarum polybotrys Hand.-Mazz., a popular phytotherapeutic medicine in China, that has attracted attention due to their antitumor activity [71, 72] and synthesized sulfated derivative (RHPS). They observed that the RHPS showed greater antitumor activity than the native polysaccharide $(p<0.05)$ in assays in vitro with epithelial cell lines of human lung adenocarcinoma (A549) and human gastric cancer (BGC-823), confirming the action of grouping sulfate.

Antitumor substances have been identified in several species of mushrooms, the polysaccharides are better known and potent antitumor and immunomodulatory properties [73]. Compared with proteins and nucleic acids, polysaccharides offer greater capacity to carry biological information, because of its potential for structural variability [74]. Such variability gives flexibility in accurate regulatory mechanisms multiple cell-cell interactions in complex organisms.

The bioactivity of Basidiomycetes mushrooms was confirmed first by Lucas in 1957, when he isolated a substance from Boletus edulis which showed a significant inhibitory effect against tumor cells of sarcoma (S-180) [75]. The proposed mechanism by which mushroom polysaccharide exert antitumor effect include: 1) prevention of oncogenesis by oral administration of the polysaccharides isolated from medicinal mushrooms (cancer preventive activity); 2) enhancement of immunity against tumors bearing (activity immunoenhancement), and 3) direct antitumor activity to induce apoptosis of tumor cells (direct inhibition of the activity of tumor) [73].

Many polysaccharides and polysaccharide conjugates have been commercialized for the clinical treatment of patients in anticancer therapy, and they schizophyllan [Fig-5], lentinan [Fig-6], grifolan, krestin (polysaccharide-peptide complex) and PSK (polysaccharide- 
protein complex) [73].

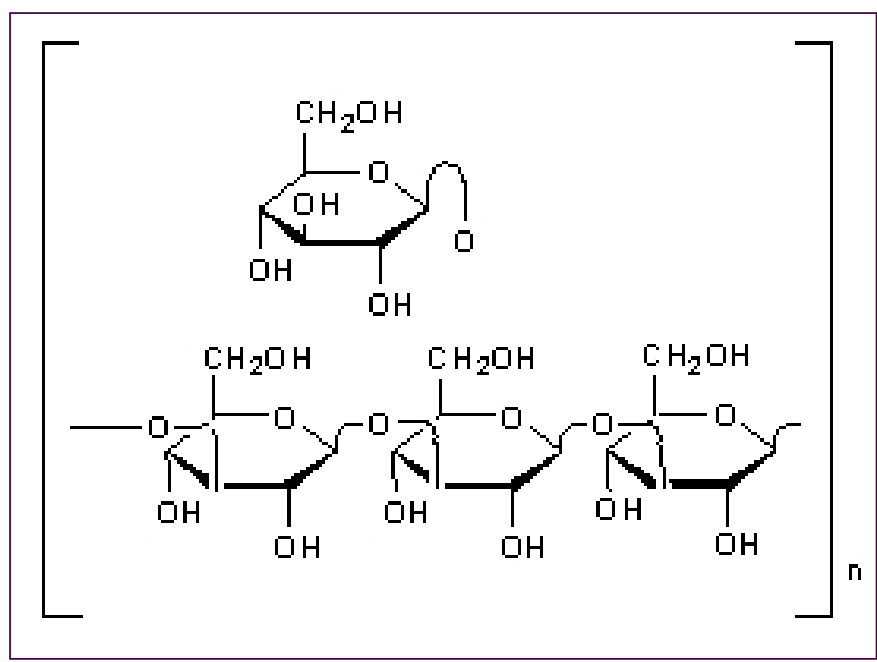

Fig. 5- Structure of schizophyllan.

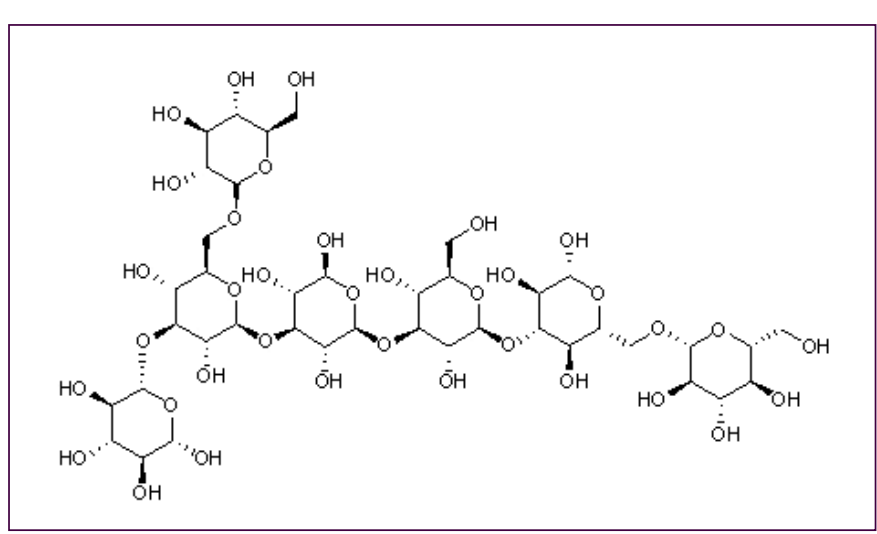

Fig. 6- Structure of lentinan.

Recently, researchers have explored compounds of marine organisms, due to their remarkable physiological activities. Thus, Sasaki and coworkers [76] isolated from the marine cyanobacterium Lyngbya sp. a new potent cytotoxic peptide bisebromoamide [Fig7], which shows antiproliferative activity at nanomolar levels.

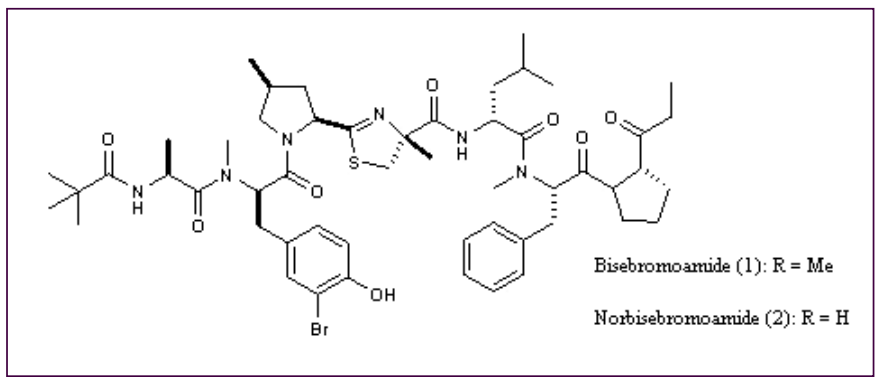

Fig. 7- Structure of bis-bromoamide.

Yokosuka and coworkers [77] isolated from the leaves of a Brazilian tree Ateleia glazioviana is a flavone glaziovianin A [Fig-8], which exhibited cytotoxicity against human promyelocytic leukemia cells (HL-60). The differential cytotoxicity standards have suggested that the mechanism of action involves the inhibition of tubulin polymerization [77]. According Ikedo and associates [78], this mechanism of action has become clinically important for drugs against breast cancer.

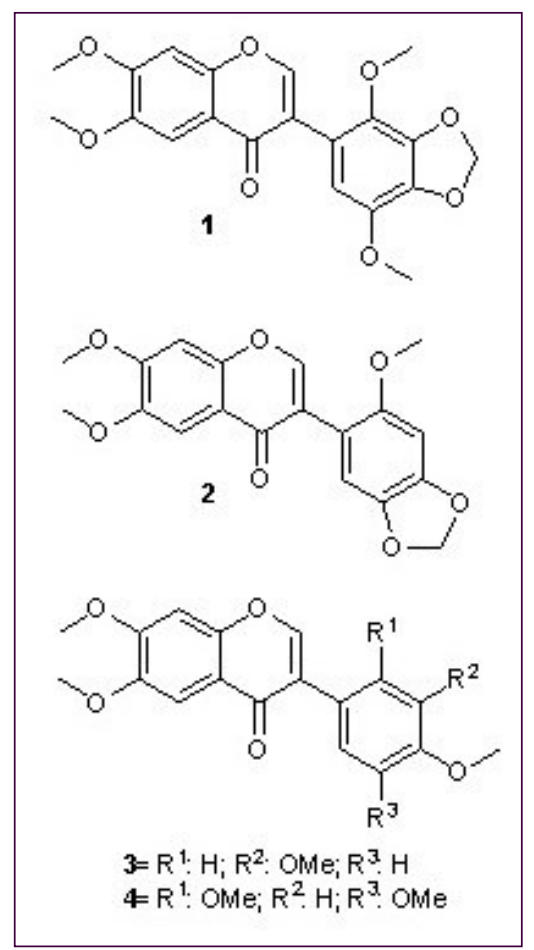

Fig. 8- Glaziovianin A (1) 6,7,20,50-tetramethoxy-30, 40methylenedioxyisoflavone; (2) 6,7,20-trimethoxy-40,50methylenedioxyisoflavone; (3) 6,7,30,40-tetramethoxyisoflavone; (4) 6,7,20,40,50-pentamethoxyisoflavone.

Smyrniotopoulos and coworkers [79] isolated from the organic extract of the red alga Sphaerococcus coronopifolius brominated diterpenes (4-16) [Fig-9], with the most active compounds were 4, 6 and 8 through the test with the cell line glioblastoma (GBM) U373 resistant to apoptosis. Compounds 4 and 6 are cytostatic agents that retard the growth of GBM U373 cells by reducing the entry in mitosis (compound 4) and increasing the duration of mitosis (compound 6 ) with an average duration of 2-3 $\mathrm{h}$ in the control condition for over $15 \mathrm{~h}$ under experimental conditions where the cells were treated for three days. In contrast, compound 8 showed cytostatic effects through indirect effects: it does not increase the duration of mitosis but induces vacuolation process, which slows down cell proliferation. This process of vacuolation may be related to both the proautophagy [80], and induction of permeability of lysosome membrane, which is related to cell death [81].

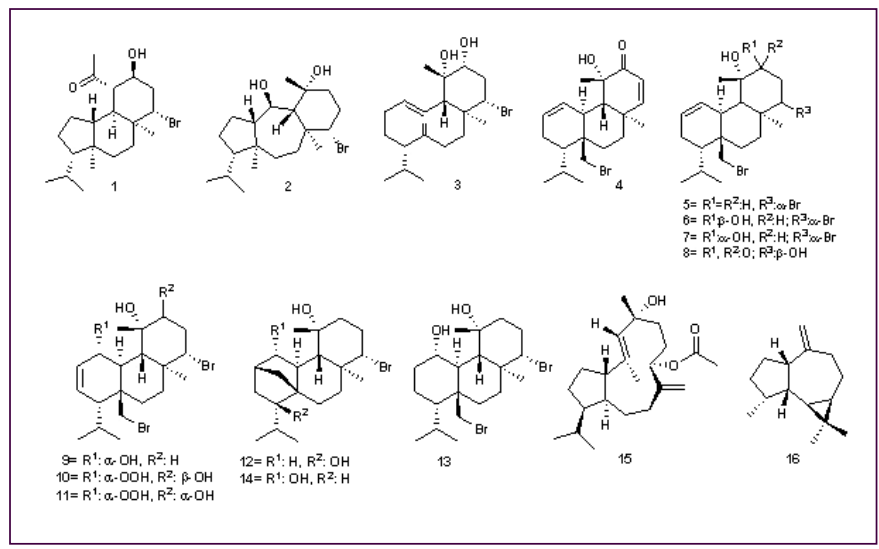

Fig. 9- Structures of brominated diterpenes isolated from red alga Sphaerococcus coronopifolius and their relative configurations. 
Animal poisons are very rich sources of biomolecules with a wide range of activities on diverse physiological systems. Among these, the snake venoms are more concentrated secretory fluids that are known, and cause a variety of biological effects [82]. Studies show that various substances found in snake venoms have some antitumor activity, as components belonging to the family of metalloproteinases, C-type lectin and disintegrins that inhibit cell migration in vitro and tumor progression in vivo by interacting specifically with some integrins in cells membranes [83]. More recently, it has been demonstrated that crotamine, protein isolated from the venom of the South American rattle snake Crotalus durissusterrificus, targets tumor tissue in vivo activating the executive enzyme caspase and triggers a lethal calcium-dependent pathway, modifying mitochondrial membrane potential in cultured cells [84].

Lately, it has been reported in Cuba using an alternative drug for the treatment of cancer, from the venom of a scorpion endemic to that country Rhopalurus junceus, popularly called "blue scorpion", which dilute the poison known by the brand "Escozul" is administered to patients [85].

The combination of natural products and commercialized drugs has also been explored recently. Sadzuka and coworkers [86] extracted curcumin (diferuloylmethane), a phenolic compound from the root of the plant Curcuma longa Linn popularly known as Indian Saffron, which is used as a coloring and flavoring in foods [Fig-10]. Curcumin was combined with chemotherapeutic drug doxorubicin (DOX), which alone did not reduce tumor weight and after the association was observed $56.5 \%$ reduction in tumor weight $(p<0.05)$ compared to the group control. This combination enhanced apoptosis, decreased cell viability, and suppresses the activation of caspase-3, 8 and -9 compared with the action of chemotherapeutic alone.

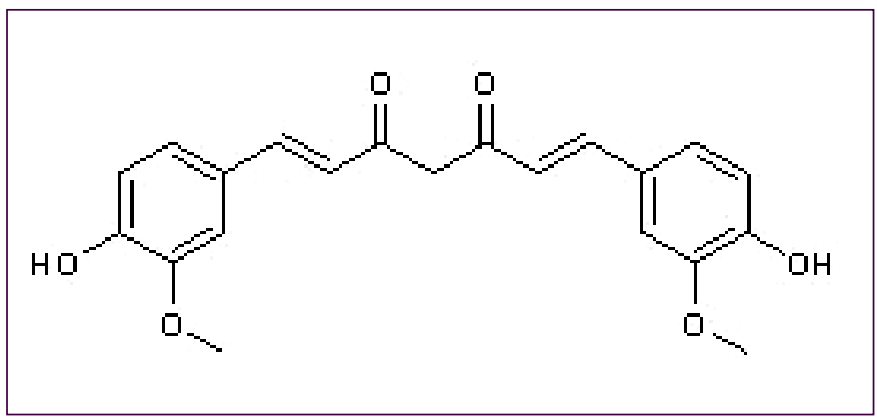

Fig. 10- Structure of curcumin.

\section{Heterocycles in Cancer Treatment}

Heterocyclic molecules are cyclic molecules, aromatic or not, having in its constitution, in addition to carbon atoms, one or more atoms of oxygen, nitrogen or sulfur. Heretocycles have a long history in medicinal chemistry. Several compounds containing heterocyclic rings are being studied to treat different types of cancer, such as the monastrol and ispinesib. While others are used by patients as methotrexate used as anticancer agent, Gemzar ${ }^{\circledR}$ (gemcitabine) for the treatment of lung and pancreas, and Temodal ${ }^{\circledR}$ (temozolomide) for the treatment of glioma and melanoma [87].

\section{Monastrol}

Recent research involving dihydropyrimidinones (six-membered rings with two nitrogen atoms, in positions 3 and 4) that have a resemblance to the structure of nucleic acid bases found in RNA showed the monastrol (5-ethoxycarbonyl-6-methyl-4-(3-
hydroxyphenyl)-3,4-dihiropirimidina-2(1H)-thione) as a promising anticancer agent, since it acts as an inhibitor of Eg5 kinesin [88]. The kinesin Eg5 is a protein that plays a crucial role in the generation of bipolar spindle, which leads to disruption of mitosis and subsequently cell death by apoptosis [89].

The monastrol had anticancer effect especially in mammalian cells, but similar dihydropyrimidinones being tested in order to find new functions in the body such heterocycles [88]. Soumyanarayanan and coworkers [88] synthesized a series of similar molecules to monastrol, starting Biginelli reaction to synthesis of dihydropyrimidinones, which was subsequently hydrolyzed and were finally subjected to a coupling reaction with cyclic amines [Fig-11].

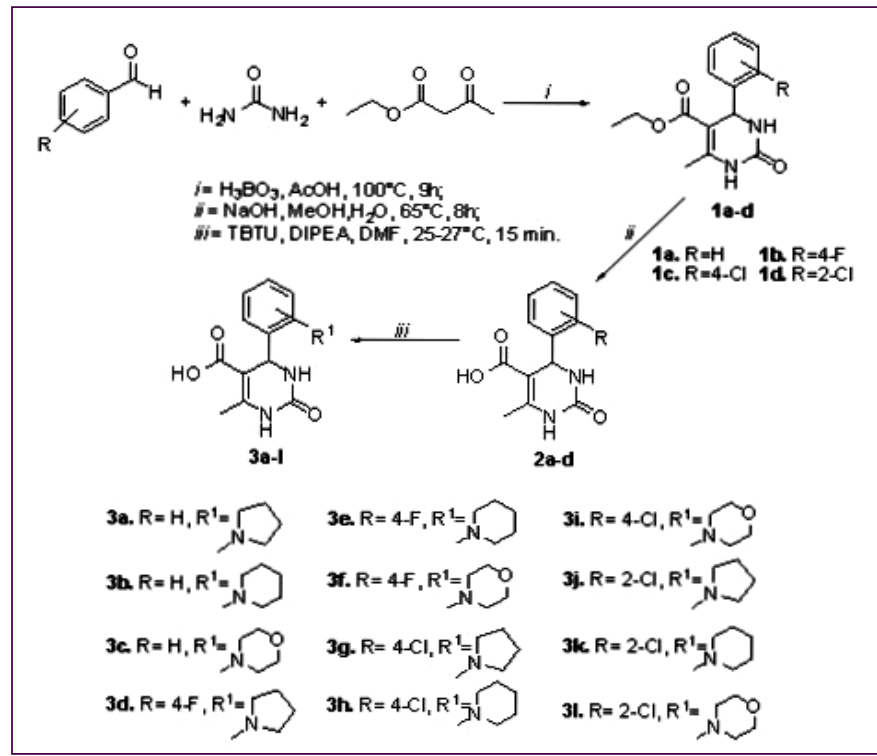

Fig. 11- Synthesis of monastrol analogues.

Subsequently, these molecules were tested for anticancer activity in hepatocellular carcinoma cells (HepG2), and human epithelial carcinoma (HeLa). The best results for HepG2 were substances $3 g$ and $3 \mathrm{~h}$, with $\mathrm{IC}_{50} 124.46$ and $120.62 \mathrm{mg} \cdot \mathrm{mL}^{-1}$, respectively [88]. Substances in these studies structure-activity relationships and molecular modeling were also performed.

\section{Ispinesib}

The Ispinesib [Fig-12] is an inhibitor of KSP kinesin Eg5 as monastrol [90].<smiles>Cc1ccc(C(=O)N(CCCN)C(c2nc3cc(Cl)ccc3c(=O)[nH]2)C(C)C)cc1</smiles>

(R)-N-(3-aminopropy)-N-(1-(7-chloro-4-0x0-3,4dihydroquinazolin-2-y)-2-methy(propyl)-4-methylbenzamide

Fig. 12- Ispinesib structure.

Purcell and coworkers [91] studied the potential growth inhibition of breast cancer through the use of Ispinesib. Through experiments 
involving several cell lines of breast cancer in grafts in vitro and in vivo, was also tested the ability of Ispinesib to increase the antitumor activity of therapies approved. According to the observed results was perceived antiproliferative activity against 53 strains of cells in vitro and tumor regression in vivo as well as combination therapy, showing that this drug may help treat cancer [91].

Structures of anticancer drugs that act during DNA synthesis are related [Fig-13].

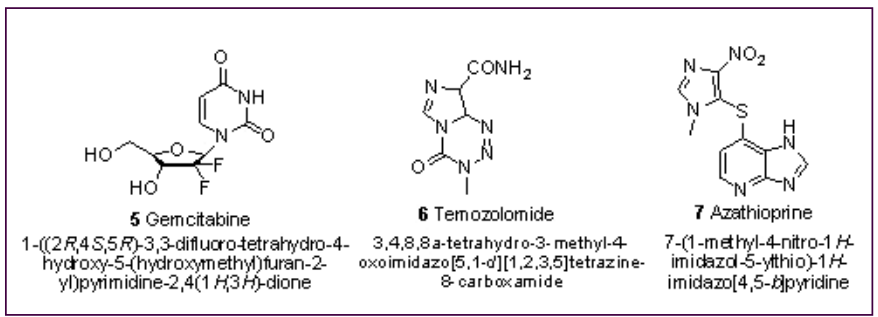

Fig. 13- Structures of drugs that interfere with DNA synthesis.

\section{Gemcitabine}

Gemcitabine (5) [Fig-13], is a nucleoside analogue that replaces one of the building blocks of nucleic acids, at this particular condition cytidine, in DNA replication. The process contains tumor growth resulting in apoptose [92].

\section{Temozolomide}

Temozolomide (6) [Fig-13], is an agent imidazotetracenic alkylating with antitumor activity, which undergoes rapid chemical conversion at physiological $\mathrm{pH}$ in systemic circulation, that forms the active compound monomethyltriazeno-imidazole-carboxamide (MTIC). The cytotoxicity of MTIC is mainly due to DNA methylation at the $\mathrm{O} 6$ and $\mathrm{N} 7$ guanine positions, this leads to inhibition of DNA replication and consequently, cell death [93].

\section{Azathioprine}

Azathioprine (7) is employed to treat leukemia, metabolized to 6mercaptopurine (6-MP). It is a purine analog, which has cytotoxic effects on lymphocytes, inhibits the synthesis of ribonucleotides and NK cells [94]. In addition to these drugs, we can still highlight the methotrexate and 5-fluorouracyl [Fig-14].

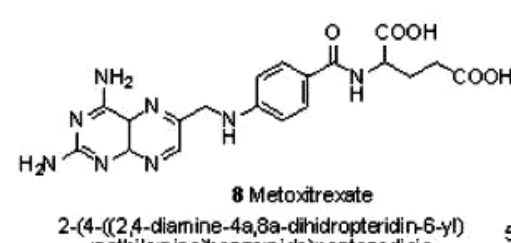

2-(4-((2,4-diamine-4a,8a-dihidropteridin-6-yl)
methilamine benzamido)pentanedicic

Fig. 14- Drugs used in cancer treatment.

\section{Methotrexate}

The methotrexate (8) [Fig-14] is largely established as anticancer drug in high doses and immunosuppressive agents when administered in smaller doses. This drug is an antimetabolite with similar structure to folic acid, which competitively inhibits the activity of the enzyme dihydrofolate reductase being considered chemotherapeutic specific at the synthesis phase of the cell cycle, especially at exponential cell growth, as tumor [95].

\section{Fluourouracyl}

Fluorouracyl (9), pyrimidine analogue [Fig-14], is used for the treatment of solid tumors, acting as an uracil antimetabolite. In organism, prevents the biosynthesis of pyrimidine nucleotides interfering with vital cellular activity, and enzymatically converted to the active deoxynucleotide, then interfere with DNA synthesis [96].

\section{Nanotechnology Applied to Drugs}

Systemic administration of most chemotherapeutic drugs (e.g., $\mathrm{Taxo}^{\circledR}$ ) can only deliver a limited amount of drug to the tumor site and commonly produces severe side effects at high doses, which limits its therapeutic index [97]. Therefore, localized delivery of antitumor drugs can increase the long-term regional drug concentration and promote the absence of systemic toxicity [98]. At this perspective, nanotechnology has received great attention due to its important clinical application on the treatment of various diseases, including cancer. Nanoparticles with diameters from 1 to $100 \mathrm{~nm}$ are employed in nanomedicine to encapsulate drugs and target them to tumors [99].

The nanoparticle enhances local drug delivery efficiency to the tumors via molecular targeting to cells expressing cancer biomarkers, entrapment in leaky tumor vasculature or magnetic targeting. Another important feature is the improvement of localization by using triggered release in tumors by optical, chemical or thermal signals. Thus, to optimize these mechanisms of nanoparticle drug delivery, it is essential to image where the nanoparticles distribute and how rapidly they release their drug payloads [100].

Promising therapeutic paradigms are coming up as multifunctional or theranostic agents, those provide attractive vehicles for both therapeutic agents and image. Nanosystems are used to diagnostic, monitoring therapeutic response and in specific targeted drug therapy. Nanomolecules present well-developed surface nature, so they are easy to anchor with multifunctional groups [101]. Currently, biodegradable polymers or lipid-based colloids are the only drug vehicles approved for clinical use, among them micelles, solid lipid nanoparticles, lipid nanocapsules, nanoemulsions and suspensions and lipoproteins which improve the in vitro dissolution velocity and in vivo efficiency of drugs with poor water solubility [102].

Recent study showed that nano-sized thermo-sensitive amphiphilic micelles have the potential to be used as a drug carrier in the chemotherapy of cholangiocarcinoma, due to their passive targeting and thermo-induced active release mechanisms [103]. Nanoparticle generated heat melts the membrane of a complex nanocarrier or the linker between the nanoparticle and therapeutic molecule within minutes or less, and releases its therapeutic payload [104]. Wang and coworkers [105] reported that using cyclic peptide-based supramolecular structures as nanocarriers is a feasible and a potential solution for drug delivery to resistant tumor cells in tests with doxorubicin in human breast cancer (MCF-7/ADR).

Magnetic nanoparticles have been reported due to their peculiar characteristics and biomedical applications, acting as diagnostic molecular imaging agents and with therapeutic properties for different types of drug carriers [106]. Others nanoparticles such as iron oxide, quantum dots (QD), silica nanoparticles, carbon nanotubes (CNTs), gold, dendrimer and graphene have been investigated as multifunctional nanoparticles [101].

The inhibition of cancer cell metastasis by graphene and graphene oxide might provide new insights into specific cancer treatment, 
once that exposure of cells to graphene led to the direct inhibition of the electron transfer chain complexes I, II, III and IV, specially by disrupting electron transfer between iron-sulfur centers. This property of graphene is due to its stronger ability to accept electrons compared to iron-sulfur clusters. The decreased electron transfer chain activity results in low production of ATP and subsequent impairment of F-actin cytoskeleton organization, that is central for the invasion and migration of metastatic cancer cells [107].

\section{Conclusion}

The understanding of the complex regulatory system involved in tumorigenesis is fundamental to the elucidation of target pathways for action new anticancer drugs. Numerous drugs used today are synthesized from biomolecules discovered both plant and animal origin, as noted along the review. Ongoing research continues to discover new potentially active biomolecules in order to optimize the therapeutic effect mainly aimed at reducing side effects.

\section{List of Acronyms and Definitions Used}

APCs: Antigen Presenting Cells: dendritic cells that play a critical role in the regulation of the adaptive immune response.

EGFR: Epidermal growth factor receptors.

Hh: Hedgehog signaling.

IGF: Insulin-like Growth Factor.

JNK: c-Jun N-terminal Kinase.

KRAS: Protein involved Primarily in Regulating Cell Division.

LC3: microtubule associated process 1 Light Chain 3 - mammalian autophagy protein.

NK: Natural Killer.

PI3K: Phosphatidylinositol 3-Kinase

PUMA: Protein that belongs to the BCL2 family and that promotes mitochondrial outer membrane permeabilization and apoptosis.

TGF- $\beta$ : Transforming growth factor-beta: potent immunosuppressor, which perturbation of its signaling is linked to autoimmunity, inflammation and cancer.

TNF: Tumor Necrosis Factors: multifunctional group proinflammatory cytokines which activate signaling pathways for cell survival, apoptosis, inflammatory responses, and cellular differentiation.

VEGF: Vascular endothelial growth factor.

\section{Conflicts of Interest: None declared.}

\section{References}

[1] Greaves M. (2007) Nat. Rev. Cancer, 7(3), 213-221.

[2] David A.R. \& Zimmerman M.R. (2010) Nat. Rev. Cancer, 10 (10), 728-733.

[3] Papavramidou N., Papavramidis T. \& Demetriou T. (2010) Ann. Surg. Oncol., 17(3), 665-667.

[4] Nobili S., Lippi D., Witort E., Donnini M., Bausi L., Minia E. \& Capaccioli S. (2009) Pharmacol. Res., 59(6), 365-378.

[5] Meesat R., Belmouaddine H., Allard J.F., Tanguay-Renaud C., Lemay R., Brastaviceanu T., Tremblay L., Paquette B., Wagner J.R., Jay-Gerin J.P., Lepage M., Huels M.A. \& Houde D. (2012) Proceedings of the National Academy of Sciences, 109(38), E2508-E2513.
[6] Morrison W.B. (2010) J. Vet. Intern. Med., 24(6), 1249-1262.

[7] Martens E.A., Kostadinov R., Maley C.C., Hallatschek O. (2011) New J. Phys., 13(1), 1-22.

[8] Huang S. \& Ingber D.E. (2007) Breast Disease, 26(1), 27-54.

[9] Urata S., Yoshida M., Ebihara Y. \& Asakage T. (2013) Auris Nasus Larynx, 40(6), 577-580.

[10]Hanahan D. \& Weinberg R.A. (2000) Cell, 100(1), 57-70.

[11]Hanahan D. \& Weinberg R.A. (2011) Cell, 144(5), 646-674.

[12]Lopes A.A., Oliveira A.M. \& Prado C.B.C. (2002) Rev. Biol. Ciênc. Terra, 2(2), 1-7.

[13]Filippi I., Naldini A. \& Carraro F. (2011) Curr. Med. Chem., 18 (19), 2885-2892.

[14]Cho W.C.S. (2012) Expert Opin. Ther. Targets, 16(1), 1-3.

[15]Jones S., Zhang X., Parsons D.W., Lin J.C.H., Leary R.J., Angenendt P., Mankoo P., Carter H., Kamiyama H., Jimeno A., Hong S.M., Fu B., Lin M.T., Calhoun E.S., Kamiyama M., Walter K., Nikolskaya T., Nikolsky Y., Hartigan J., Smith D.R., Hidalgo M., Leach S.D., Klein A.P., Jaffee E.M., Goggins M., Maitra A., lacobuzio-Donahue C., Eshleman J.R., Kern S.E., Hruban R.H., Karchin R., Papadopoulos N., Parmigiani G., Vogelstein B., Velculescu V.E. \& Kinzler K.W. (2008) Science, 321(5897), 1801-1806.

[16]Takebe N., Warren R.Q. \& Ivy S.P. (2011) Breast Cancer Res., 13(3), 211-222.

[17]de Luca A., Maiello M.R., D’Alessio A., Pergameno M. \& Normanno N. (2012) Expert Opin. Ther. Targets, 16(1), 17-27.

[18]Shin S.W., Seo C.Y., Han H., Han J.Y., Jeong J.S., Kwak J.Y. \& Park J.I. (2009) Clin. Cancer Res., 15(17), 5414-5425.

[19]Harris P.J., Speranza G. \& Ullmann C.D. (2012) Expert Opin. Ther. Targets, 16(1), 131-45.

[20]Onishi H. \& Katano M. (2011) Cancer Sci., 2011, 102(10), 1756 -1760 .

[21]Razumilava N. \& Gores G.J. (2013) J. Hepatol., 58(6), 12441245.

[22]Krishnan V., Bryant H.U. \& MacDougald O.A. (2006) J. Clin. Invest., 116(5), 1202-1209.

[23]Néstor T., Masckauchán H. \& Kitajewski J. (2006) Physiology, 21(3), 181-188.

[24]Chien A.J., Conrad W.H. \& Moon R.T. (2009) J. Invest. Dermat., 129(7), 1614-1627.

[25]Maruotti N., Corrado A., Neve A. \& Cantatore F.P. (2013) J. Cell. Physiol., 228(7), 1428-1432.

[26]Rosenbluh J., Nijhawan D., Cox A.G., Li X., Neal J.T., Schafer E.J., Zack T.I., Wang X., Tsherniak A., Schinzel A.C., Shao D.D., Schumacher S.E., Weir B.A., Vazquez F., Cowley G.S., Root D.E., Mesirov J.P., Beroukhim R., Kuo C.J., Goessling W. \& Hahn W.C. (2012) Cell, 151(7), 1457-1473.

[27]Gui S., Yuan G., Wang L., Zhou L., Xue Y., Yu Y., Zhang J., Zhang M., Yang Y. \& Wang D.W. (2013) J. Cell. Biochem., 114 (7), 1488-1497.

[28]Villa-Morales M. \& Fernández-Piqueras J. (2012) Expert Opin. Ther. Targets, 16(1), 85-101.

[29]Waldner M.J. \& Neurath M.F. (2012) Expert Opin.Ther. Targets, 16(1), 05-13. 
[30]Seshacharyulu P., Ponnusamy M.P., Haridas D., Jain M., Ganti A.K. \& Batra S.K. (2012) Expert Opin. Ther. Targets, 2012, 16 (1), 15-31.

[31]Remon J., Morán T., Majem M., Reguart N., Dalmau E., Márquez-Medina D. \& Lianes P. (2014) Cancer Treat. Rev., 40(1), 93-101.

[32]Huang X., Li Y., Zhang J., Xu Y., Tian Y. \& Ma K. (2013) J. Cell. Biochem., 114(7), 1616-1624.

[33]Tognon C.E. \& Sorensen P.H.B. (2012) Expert Opin. Ther. Targets, 16(1), 33-48.

[34]Trajkovic-Arsic M., Kalideris E. \& Siveke J.T. (2013) J. Mol. Endocrinol., 50(3), R67-R74.

[35]Santarpia L., Lippman S.M. \& El-Naggar A.K. (2012) Expert Opin. Ther. Targets, 16(1), 103-119.

[36]Peng Z., Xue B., Kurgan L. \& Uversky V.N. (2013) Cell Death Differ., 20(9), 1-11.

[37]Sanz A.B., Sanchez-Niño M.D., Izquierdo M.C., GonzalezEspinoza L., Ucero A.C., Poveda J., Ruiz-Andres O., RuizOrtega M., Selgas R., Egido J. \& Ortiz A. (2014) Int. Rev. Immunol., 33(1), 09-22.

[38]Ouyang L., Shi Z., Zhao S., Wang F.T., Zhou T.T., Liu B. \& Bao J.K. (2012) Cell Prolif., 45(6), 487-498.

[39]Galluzzi L., Maiuri M.C., Vitale I., Zischka H., Castedo M., Zitvogel L. \& Kroemer G. (2007) Cell Death Differ., 14(7), 12371243.

[40]Bialik S., Zalckvar E., Ber Y., Rubinstein A.D. \& Kimchi A. (2010) Trends Biochem. Sci., 35(10), 556-564.

[41]Vousden K.H. \& Lane D.P. (2007) Nat. Rev. Mol. Cell Biol., 8 (4), 275-283.

[42]Folkman J. (2007) Nat. Rev. Drug Discov., 6(4), 273-286.

[43]Sakurai T., Okumura H., Matsumoto M., Uchikado Y., Setoyama T., Omoto I., Owaki T., Maemura K., Ishigami S. \& Natsugoe S. (2013) Med. Oncol., 30(4), 701.

[44]Korpis K., Weber F., Brune S., Wünsch B. \& Bednarski P.J. (2014) Bioorg. Med. Chem., 22(1), 221-233.

[45]Fujii S., Mitsunaga S., Yamazaki M., Hasebe T., Ishii G., Kojima M., Kinoshita T., Ueno T., Esumi H. \& Ochiai A. (2008) Cancer Sci., 99(9), 1813-1819.

[46]Yoshioka A., Miyata H., Doki Y., Yamasaki M., Sohma I., Gotoh K., Takiguchi S., Fujiwara Y., Uchiyama Y. \& Monden M. (2008) Int. J. Oncol., 33(3), 461-468.

[47]Czaja M.J. (2011) Gastroenterology, 140(7), 1895-1908.

[48]Cui J., Gong Z. \& Shen H.M. (2013) Biochim. Biophys. Acta, 1836(1), 15-26.

[49]Amaravadi R.K., Lippincott-Schwartz J., Yin X.M., Weiss W.A., Takebe N., Timmer W., DiPaola R.S., Lotze M.T. \& White E. (2011) Clin. Cancer Res., 17(4), 654-666.

[50]Vandenabeele P., Galluzzi L., Vanden B.T. \& Kroemer G. (2010) Nat. Rev. Mol. Cell Biol., 11(10), 700-714.

[51]Fink S.L. \& Cookson B.T. (2006) Cell. Microbiol., 8(11), 18121825.

[52]Stegh A.H. (2012) Expert Opin. Ther. Targets, 16(1), 67-83.

[53]Begley J. \& Ribas A. (2008) Clin. Cancer Res., 14(14), 43854391.
[54]Dunn G.P., Old L.J. \& Schreiber R.D. (2004) Immunity, 21(2), 137-148.

[55]Silva J.M., Videira M., Gaspar R., Préat V. \& Florindo H.F. (2013) J. Controll. Release, 168(2), 179-199.

[56]Koudougou C., Bonneville M., Matysiak-Budnik T. \& Touchefeu Y. (2013) Aliment. Pharmacol. Ther., 38(1), 03-15.

[57]Rachagani S., Torres M.P., Moniaux N. \& Batra S.K. (2009) BioFactors, 35(6), 509-527.

[58]Kessenbrock K., Plaks V. \& Werb Z. (2010) Cell, 141(1), 52-67.

[59]Gocheva V., Wang H.W., Gadea B.B., Shree T., Hunter K.E., Garfall A.L., Berman T. \& Joyce J.A. (2010) Genes Dev., 24(3), 241-255.

[60]Grivennikov S.I., Greten F.R. \& Karin M. (2010) Cell, 140(6), 883-899.

[61]Butler M.S. (2008) Nat. Prod. Rep., 25(3), 475-561.

[62]Xu M., Takanashi M., Oikawa K., Nishi H., Isaka K., Yoshimoto T., Ohyashiki J. \& Kuroda M. (2012) Cancer Sci., 103(4), 821827.

[63]Holton R.A., Kim H.B., Somoza C., Liang F., Biediger R.J., Boatman P.D., Shindo M., Smith C.C. \& Kim S. (1994) Journal of the American Chemical Society, 116(4), 1599-1600.

[64]Sathisha M.P., Budagumpi S., Kulkarni N.V., Kurdekar G.S., Revankar V.K. \& Pai K.S.R. (2010) Eur. J. Med. Chem., 45(1), 106-113.

[65]Sears P. \& Wong C.H. (1999) Angew. Chemie, 111(16), 24462471.

[66]Rouquayrol M., Gaucher W., Greiner J., Aubertin A.M., Vierling P. \& Guedj R. (2001) Carbohydr. Res., 336(3), 161-180.

[67]Geren R.I., Greenberg N.H., Mac Donald M.M., Schumacher A.M. \& Abbot B.J. (1972) Cancer Chemoth. Rep., 3(1), 01-102.

[68]Wei D., Wei Y., Cheng W. \& Zhang L. (2012) Int. J. Biol. Macromol., 51(4), 471-476.

[69]Lu Y., Wang D.Y., Hu Y.L., Huang X.Y. \& Wang J.M. (2008) Carbohydr. Polym., 71(2), 180-186.

[70]Nie X.H., Shi B.J., Ding Y.T. \& Tao W.Y. (2006) Int. J. Biol. Macromol., 39(4-5), 228-233.

[71]Shon Y.H., Kim J.H. \& Nam K.S. (2002) Biol. Pharm. Bull., 25 (1), $77-80$.

[72]Song Z.H., Ji Z.N. \& Lo C.K. (2004) Planta Med., 70(12), 12221227

[73]Zhang M., Cui S.W., Cheung P.C.K. \& Wang Q. (2007) Trends Food Sci. Tech., 18(1), 04-19.

[74]Leung S. \& Romanowski M. (2012) Theranostics, 2(10), 10201036.

[75]Ohno N. (2005) Int. J. Med. Mushrooms, 7(1-2), 167-188.

[76]Lucas E.H. (1957) Antibiot. Chemother., 7(1), 1-15.

[77]Sasaki H., Teruya T., Fukazawa H. \& Suenaga K. (2011) Tetrahedron, 67(5), 990-994.

[78]Yokosuka A., Haraguchi M., Usui T., Kazami S., Osada H., Yamori T., Mimaki Y. \& Glaziovianin A. (2007) Bioorg. Med. Chem. Lett., 17(11), 3091-3094.

[79]Ikedo A., Hayakawa I., Usui T., Kazami S., Osada H. \& Kigoshi H. (2010) Bioorg. Med. Chem. Lett., 20(18), 5402-5404. 
[80]Smyrniotopoulos V., Vagias C., Bruyère C., Lamoral-Theys D., Kiss R. \& Roussis V. (2010) Bioorg. Med. Chem., 18(3), 13211330.

[81]Lefranc F., Mijatovic T., Kondo Y., Sauvage S., Roland I., Debeir O., Krstic D., Vasic V., Gailly P., Kondo S., Blanco G. \& Kiss R. (2008) Neurosurgery, 62(1), 211-222.

[82]Mijatovic T., Mathieu V., Gaussin J.F., De Nève N., Ribaucour F., Van Quaquebeke E., Dumont P., Darro F. \& Kiss R. (2006) Neoplasia, 8(5), 402-412.

[83]Stocker K.F. (1990) Medical Use of Snake Venom Proteins, CRC press, New York, 34-50.

[84]Kamiguti A.S., Hay C.R.M., Theakston R.G.D. \& Zuzel M. (1996) Toxicon, 34(6), 627-642.

[85]Nascimento F.D., Sancey L., Pereira A., Rome C., Oliveira V., Oliveira E.B., Nader H.B., Yamane T., Kerkis I., Tersariol I.L., Coll J.L. \& Hayashi M.A. (2011) Molecular Pharmaceutics, 9(2), 211-221.

[86]García-Gómez B.I., Coronas F.I., Restano-Cassulini R., Rodríguez R.R. \& Possani L.D. (2011) Toxicon, 58(1), 18-27.

[87]Sadzuka Y., Nagamine M., Toyooka T., Ibuki Y. \& Sonobe T. (2012) Int. J. Pharm., 432(1-2), 42-49.

[88]Joule J.A. \& Mills K. (2007) Heterocyclic Chemistry at a Glance, Oxford: Blackwell Publishing, 160.

[89]Soumyanarayanan U., Bhat V.G., Kar S.S. \& Mathew J.A. (2012) Org. Med. Chem. Lett., 2(1), 23.

[90]DeBonis S., Skoufias D.A. \& Lebeau L. (2004) Mol. Cancer Ther., 3(9), 1079-1090.

[91]Lad L., Luo L., Carson J.D., Wood K.W., Hartman J.J., Copeland R.A. \& Sakowicz R. (2008) Biochemistry, 47(11), 3576-3585.

[92]Purcell J.W., Davis J., Reddy M., Martin S., Samayoa K., Vo H., Thomsen K., Bean P., Kuo W.L., Ziyad S., Billig J., Feiler H.S., Gray J.W., Wood K.W. \& Cases S. (2010) Clin. Cancer Res., 16 (2), 566-576.

[93]Candelaria M., de la Cruz-Hernandez E., Taja-Chayeb L., Perez -Cardenas E., Trejo-Becerril C., Gonzalez-Fierro A., ChavezBlanco A., Soto-Reyes E., Dominguez G., Trujillo J.E., DiazChavez J. \& Duenas-Gonzalez A. (2012) PLoSONE, 7(3), e29181.

[94]Szeliga M., Zgrzywa A., Michelwska M.O., Albrecht J. (2012) J. Neurochem., 123(3), 428-436.

[95]Shih D.Q., Nguyen M., Zheng L., Ibanez P., Mei L., Kwan L.Y., Bradford K., Ting C., Targan S.R. \& Vasiliauskas E.A. (2012) Aliment. Pharm. Therap., 36(5), 449-458.

[96]Balaji P., Thirumal M., Gowri R., Divya V. \& Vadivelan R. (2012) Int. J. Pharm. Chem. Biol. Sci., 2(4), 464-471.

[97]Ligabue A., Marverti G., Liebl U. \& Myllykallio H. (2012) PLoSONE, 7(10), e47318.

[98]Safavy A. (2008) Curr. Drug Deliv., 5(1) 42-54.

[99]Lin Z., Gao W., Hu H., Ma K., He B., Dai W., Wang X., Wang J., Zhang X. \& Zhang Q. (2014) J. Control. Release, 174, 161-170.

[100]Valetti S., Mura S., Stella B. \& Couvreur P. (2013) J. Nanobiotechnology, 11(1-S6), 1-17.

[101]Moore T., Chen H., Morrison R., Wang F., Anker J.N. \& Alexis
F. (2014) Mol. Pharmaceutics, 11(1), 24-39.

[102]Rahman M., Ahmad M.Z., Kazmi I., Akhter S., Afzal M., Gupta G., Jalees Ahmed F. \& Anwar F. (2012) Expert Opin. Drug Deliv., 9(4), 367-381.

[103]Wang Y., Zhang L., Wang Q. \& Zhang D. (2014) J. Biomed. Nanotechnol., 10(4), 543-558.

[104]Wang X., Li S., Wan Z., Quan Z. \& Tan Q. (2014) Int. J. Pharm., 463(1), 81-88.

[105]Leung S.J. \& Romanowski M. (2012) Theranostics, 2(10), 1020-1036.

[106]Wang Y., Yi S., Sun L., Huang Y., Lenaghan S.C. \& Zhang M. (2014) J. Biomed. Nanotechnol., 10(3), 445-454.

[107]Sivakumar B., Aswathy R.G., Sreejith R., Nagaoka Y. \& Iwai S. (2014) J. Biomed. Nanotechnol., 10(6), 885-899

[108]Zhou H., Zhang B., Zheng J., Yu M., Zhou T., Zhao K., Jia Y., Gao X., Chen C. \& Wei T. (2014) Biomaterials, 35(5), 15971607. 